\title{
Descolamento seroso tardio e recorrente da coróide após trabeculectomia: relato de caso
}

\author{
Late and recurrent serous choroidal detachment after \\ trabeculectomy: case report
}

Nikias Alves da Silva ${ }^{1}$

Felício Aristóteles da Silva ${ }^{2}$

\section{RESUMO}

O presente caso refere-se a um paciente do sexo masculino, de 85 anos de idade, com catarata senil e glaucoma primário de ângulo aberto avançado nos dois olhos, não controlado com medicação máxima, hialose asteróide no OD e degeneração macular relacionada à idade no OE, submetido a facotrabeculectomia em ambos os olhos com mitomicina C. Diante da falência da cirurgia nos dois olhos, mesmo após lise de suturas com laser de argônio, uso de 5-fluorouracil e agulhamento, foi necessário prescrever novamente hipotensores oculares. Com a combinação fixa timolol 0,5\% + dorzolamida $2 \%$, o paciente apresentou descolamento seroso coroidiano bilateral com marcante hipotonia; e com brinzolamida $1 \%$ o quadro ocorreu apenas no olho esquerdo. Suspensos os colírios, a pressão intraocular se elevava e o descolamento da coróide regredia completamente. O agulhamento associado às aplicações de 5-fluorouracil resolveu a hipertensão ocular.

Descritores: Trabeculectomia/efeitos adversos; Mitomicina; Doenças da coróide/etiologia; Hipotensão ocular; Recidiva; Relatos de casos [Tipo de publicação]

\section{INTRODUÇÃO}

Descolamento seroso da coróide após uma cirurgia fistulante é uma ocorrência comum no pós-operatório imediato ${ }^{(1)}$, geralmente pequeno, benigno e tende à regressão espontânea. Já o descolamento seroso tardio da coróide após trabeculectomia é um evento raro. Frequentemente decorre de hipotonia consequente à filtração excessiva ou vazamento na ampola filtrante, particularmente quando é isquêmica e de parede fina, como frequentemente se observa após o uso de mitomicina C. Mais raro ainda é a ocorrência de descolamento seroso tardio da coróide após trabeculectomia, ou qualquer outra cirurgia fistulante, na ausência daquelas condições predisponentes, idiopática ou pelo uso de hipotensores oculares: tópicos e sistêmicos. Neste último caso, o quadro regride completamente pela suspensão das drogas, ou suspensão das mesmas associada à drenagem cirúrgica.

Portanto, o conhecimento desta possibilidade é fundamental para que o médico tome a única medida eficaz para resolver o problema, que é a suspensão dos hipotensores oculares.

\section{RELATO DO CASO}

ERO, sexo masculino, 85 anos de idade, pardo, portador de glaucoma primário de ângulo aberto avançado e catarata senil bilaterais, hialose asteróide intensa no olho direito (OD) e degeneração macular relacionada à idade 
no olho esquerdo (OE) com descolamento da retina (DR) seroso macular. A acuidade visual não corrigida no OD era 20/200 alcançando 20/70 com correção e no OE conta-dedos a $30 \mathrm{~cm}$, não melhorando com correção. Sua pressão intraocular (PIO) variava de 20-24 mmHg nos dois olhos com Xalatan ${ }^{\circledR}$ associado a $\operatorname{Cosopt}^{\circledR}$, que usava há dois anos, e a espessura corneana era $449 \mu$ no OD e $467 \mu$ no OE.

Foi submetido a uma facotrabeculectomia com mitomicina $\mathrm{C} 0,3 \mathrm{mg} / \mathrm{ml}$ por 3 minutos no OE. No $10^{\circ}$ dpo (dia de pósoperatório) a PIO estava em $19 \mathrm{mmHg}$, e o ponto central do retalho escleral foi seccionado, seguido de 2 agulhamentos com 5-FU (5 mg cada), e posteriormente revisão da trabeculectomia. Após um mês, a PIO era $20 \mathrm{mmHg}$ e a ampola filtrante mostrava-se translúcida e com microcistos. Foi reintroduzido Cosopt $^{\circledR} 12 / 12$ h, e dez dias após a PIO estava em 3 mmHg. O mesmo foi suspenso e receitado Maxidex ${ }^{\circledR} 3 \mathrm{x} / \mathrm{dia}$, e após sete dias a PIO subiu para $6 \mathrm{mmHg}$ com ótima ampola filtrante.

Após um mês, foi realizada facotrabeculectomia com mitomicina $\mathrm{C} 0,3 \mathrm{mg} / \mathrm{ml}$ por 4 minutos no $\mathrm{OD}$. No $3^{\circ} \mathrm{dpo}, \mathrm{PIO}$ $\mathrm{AO}=25 \mathrm{mmHg}$, e o ponto central do retalho escleral foi seccionado no OD. Não havia ampola filtrante no OD, e no $\mathrm{OE}$ havia uma ampola filtrante com microcistos. Foi receitado Co$\operatorname{sopt}^{\circledR} 12 / 12$ h para o OE. No $13^{\circ} \mathrm{dpo}, \mathrm{PIO}$ OD $=21$ e OE $=12 . \mathrm{O}$ paciente passou a usar o $\operatorname{Cosopt}^{\circledR}$ nos dois olhos. Cerca de 21 dias após iniciar o Cosopt $^{\circledR}$ nos dois olhos, PIO OD= 14 e $\mathrm{OE}=0$, olhos calmos, lente intraocular (LIO) em posição e câmara anterior profunda, inalterada. Havia no OE um descolamento seroso da coróide (Figura 1).

A combinação fixa foi suspensa no OE e receitado Maxidex $^{\circledR} 4 / 4 \mathrm{~h}+$ Atropina $^{\circledR} 1 \%$ 8/8 h. Quatorze dias após o diagnóstico do descolamento de coróide no OE, a PIO era $7 \mathrm{mmHg}$ e não havia mais descolamento. Após um mês a PIO $\mathrm{OE}=28 \mathrm{mmHg}$, e o Cosopt $\mathrm{t}^{\circledR}$ foi reintroduzido neste olho. Transcorrido dois meses e meio da facotrabeculectomia no OD, a $\mathrm{PIO}$ era $3 \mathrm{mmHg}$ e $5 \mathrm{mmHg}$ no OD e OE respectivamente. A combinação fixa foi suspensa nos dois olhos. Após 2 sema-

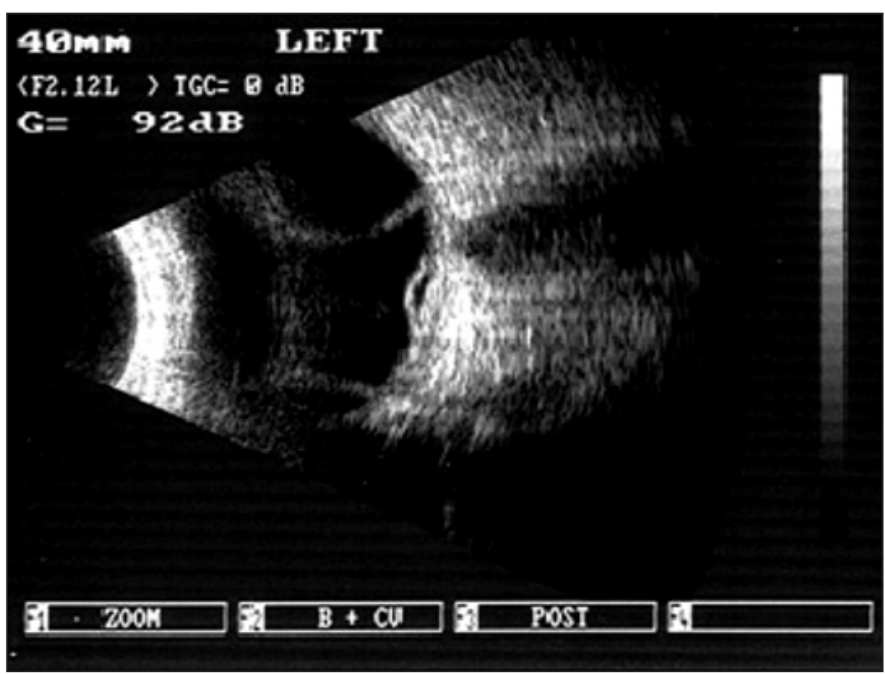

Figura 1 - OE: Descolamento seroso da coróide e da retina macular nas, $\mathrm{PIO} \mathrm{OD}=8 \mathrm{mmHg}$ e $\mathrm{OE}=2 \mathrm{mmHg}$. A ultrassonografia $\mathrm{B}$ mostrou um descolamento seroso nasal da coróide no OD (Figura 2) e OE.

Foram introduzidos os colírios de Atropina ${ }^{\circledR} 1 \%$ 12/12 h + Maxidex ${ }^{\circledR} 6 / 6 \mathrm{~h}$ nos dois olhos. Após uma semana, PIO OD= $30 \mathrm{mmHg}$ e $\mathrm{OE}=10$ e foi receitado Combigan ${ }^{\circledR}$ para o OD. A ultrassonografia B mostrou regressão do descolamento coroidiano nos dois olhos. Após 2 semanas, a PIO estava em $24 \mathrm{mmHg}$ no OD e o Combigan ${ }^{\circledR}$ foi substituído por Azopt ${ }^{\circledR} \mathrm{e}$ Alphagan $\mathrm{P}^{\circledR} 12 / 12$ h. Após 35 dias, $\mathrm{PIO} \mathrm{OD}=23$ e OE $=2 \mathrm{mmHg}$. $\mathrm{O}$ descolamento de coróide no $\mathrm{OE}$ havia reaparecido. Indagado, o paciente revelou que estava usando os colírios nos dois olhos. Com Lumingan ${ }^{\circledR}$ no OD a PIO estava em $26 \mathrm{mmHg}$.

Foi realizado um agulhamento no OD com aplicação subconjuntival per-operatória de $5 \mathrm{mg}$ de 5-FU. Desde então, a PIO neste olho se mantém entre $12-14 \mathrm{mmHg}$ sem medicação.

No OE o Azopt ${ }^{\circledR}$ foi suspenso, a PIO subiu para $30 \mathrm{mmHg}$, e o descolamento coroidiano desapareceu. Com Alphagan $\mathrm{P}^{\circledR}$ 12/12 h isolado, a PIO no OE permaneceu em $33 \mathrm{mmHg}$. Após um agulhamento com 5-FU intraoperatório, a PIO se mantém entre 13 e 14 mmHg sem medicação. No último controle havia ampolas filtrantes difusas, pouco elevadas, com abundantes microcistos e bem protegidas nos dois olhos.

\section{DISCUSSÃO}

Descolamento seroso espontâneo da coróide com hipotonia no pós-operatório tardio da trabeculectomia é uma ocorrência rara $(1,4 \%)^{(2)}$ Vela e Campbell ${ }^{(3)}$ descreveram, pela primeira vez, uma rara síndrome caracterizada por hipotonia e descolamento ciliocoroidiano após terapia farmacológica com timolol e/ou acetazolamida oral em pacientes submetidos a cirurgia fistulante 1 a 18 meses antes. Em 3 dos 4 casos, o episódio recorreu após reintrodução das drogas. Em todos os

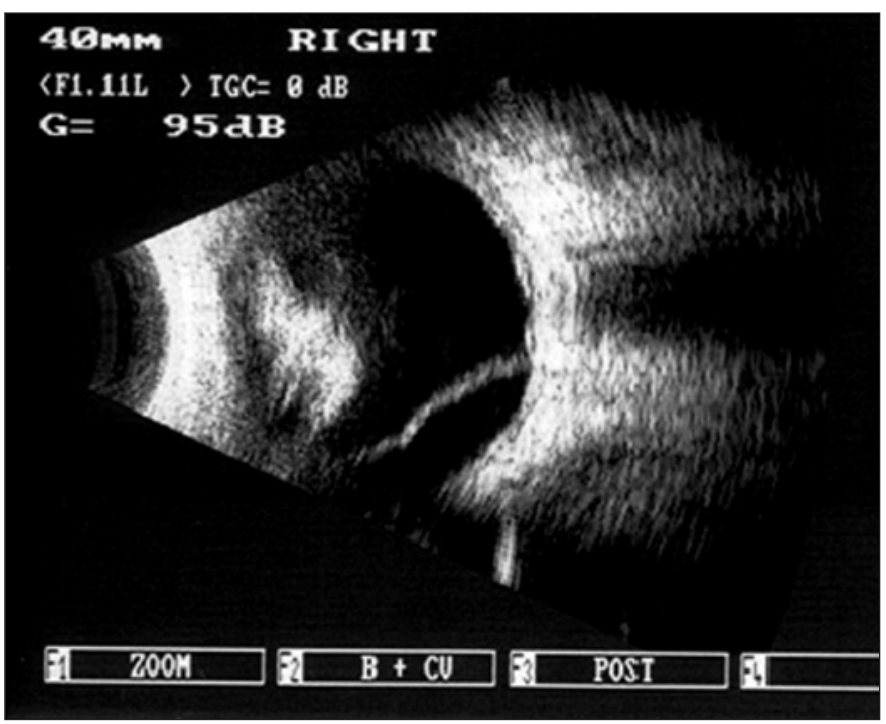

Figura 2 - OD: Descolamento seroso nasal da coróide 
casos, múltiplas drogas já haviam sido usadas antes da cirurgia, inclusive, timolol, não haviam sinais inflamatórios relevantes, a câmara anterior se mostrava rasa pelo menos na periferia e houve regressão do quadro com suspensão dos medicamentos. Outros casos têm sido publicados ${ }^{(4)}$, inclusive de ocorrência ainda mais tardia $(5 \text { anos })^{(5)}$ ou com outros hipotensores oculares (dorzolamida ${ }^{(6)}$, latanoprost ${ }^{(7)}$ ), não necessariamente supressores do humor aquoso. No presente caso, o fato se deu com Cosopt $t^{\circledR}$ nos dois olhos e Azopt ${ }^{\circledR}$ apenas no OE. A síndrome não parece depender do tipo da cirurgia, tendo sido observada inclusive em pacientes com implantes de drenagem $^{(8)}$ ou pós-facectomia. Às vezes pode haver apenas uma efusão ciliocoroidiana anterior ${ }^{(6,8)}$.

Os descolamentos ciliocoroidianos após cirurgia fistulante podem ser agudos, crônicos, inflamatório ou recorrentes. Dentre os fatores relacionados com os crônicos e recorrentes estão: idade, hipertensão arterial sistêmica, doença cardíaca arteriosclerótica, hipermetropia, terapia supressora do humor aquoso, inflamação ocular e cirurgia não protegida ${ }^{(5)}$. Neste caso, o paciente era sadio. Embora alguns autores tenham observado sinais inflamatórios significativos ${ }^{(5-6)}$, este não parece ser um componente relevante ${ }^{(3)}$. No nosso caso, o paciente nunca apresentou qualquer manifestação inflamatória. Outro fato que também chamava a atenção é que a câmara anterior estava sempre profunda, como em alguns relatos ${ }^{(6)}$, a LIO em posição e o seio camerular amplo, em contraposição a outros trabalhos em que a câmara anterior era muito rasa ${ }^{(3,7,9)}$ (Tabela 1).
Não se conhece o mecanismo exato desta síndrome. Pode ser que o corpo ciliar lesado ou previamente exposto a supressores do humor aquoso se torne supersensível e responda de maneira anômala com uma dramática redução na produção de humor aquoso quando da reintrodução dos hipotensores ocu$\operatorname{lares}^{(3)}$. Esta suscetibilidade não é permanente, porque nem sempre a reintrodução da droga volta a desencadear o fenômeno ${ }^{(3)}$. Outro fato curioso é que muitos pacientes já faziam uso há longo prazo do mesmo medicamento, sem qualquer problema ${ }^{(3)}$, como neste caso. O que é, então, que mudou nestes olhos após a cirurgia? Também não se sabe. Outro fato que nos chama a atenção é a presença de descolamento de coróide após uso de hipotensores oculares em olhos virgens de cirurgia ${ }^{(10)}$. Qual seria o mecanismo do descolamento nestes olhos?

Este parece ser o segundo caso desta síndrome publicado na literatura nacional e o primeiro da literatura mundial causado pela brinzolamida. No primeiro caso descrito na literatura nacional ${ }^{(9)}$ o descolamento ciliocoroidiano ocorreu cerca de três meses após a trabeculectomia, o paciente estava em uso de acetazolamida sistêmica, $\mathrm{PIO}=2 \mathrm{mmHg}$, e o descolamento regrediu com suspensão da medicação e drenagem supracoroidal. No presente caso o descolamento ocorreu cerca de dois meses e meio após a cirurgia filtrante no OD e quatro meses após no OE, o paciente estava em uso de Cosopt ${ }^{\circledR}$ no primeiro descolamento em ambos os olhos, Cosopt ${ }^{\circledR}$ no segundo episódio do descolamento no OE, e Azopt ${ }^{\circledR}$ e AlphaganP ${ }^{\circledR}$ no terceiro episódio deste mesmo olho, a PIO variava de

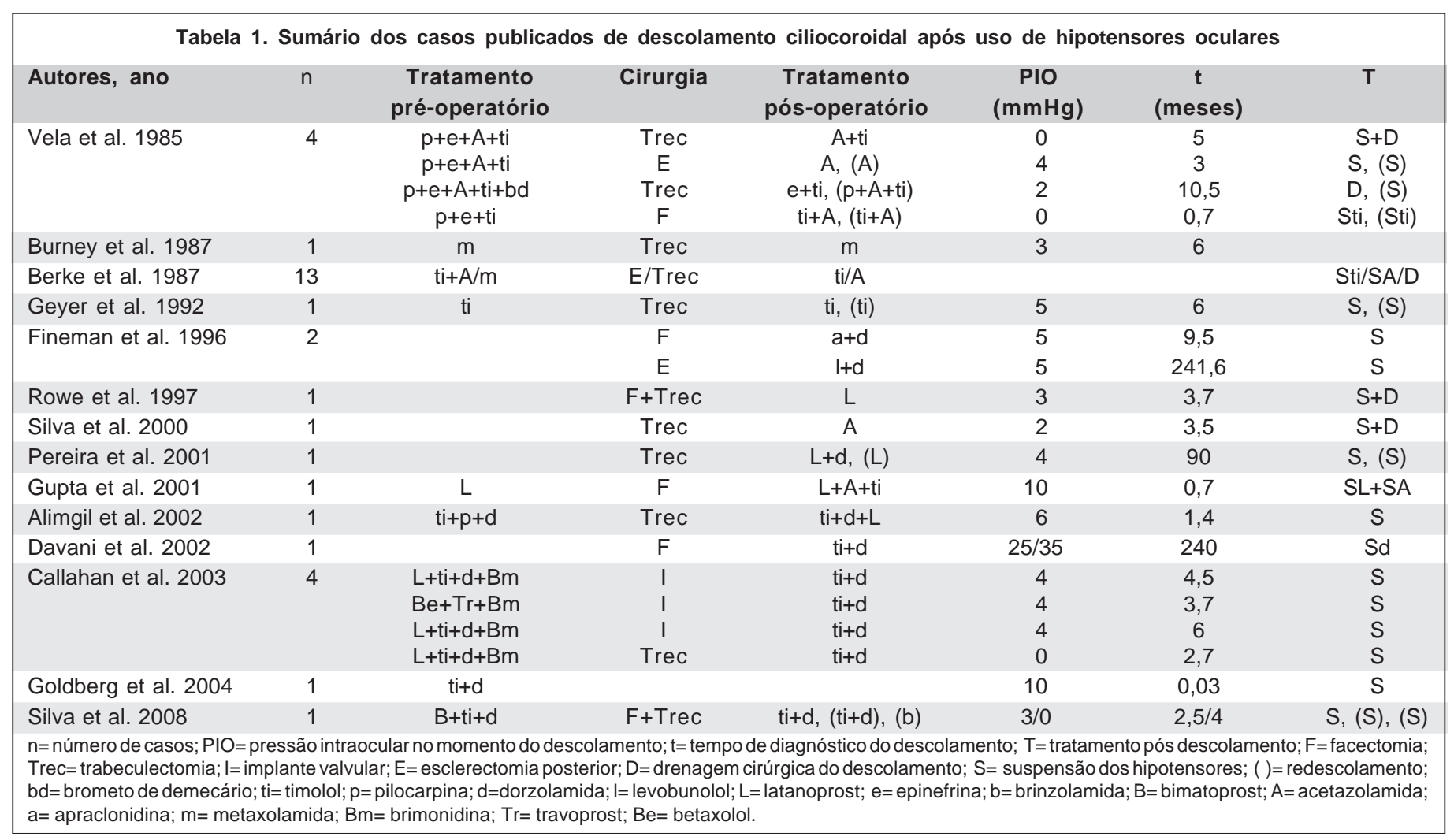




\begin{tabular}{|c|c|c|c|c|c|c|c|c|c|}
\hline \multirow[b]{2}{*}{ Olho } & \multicolumn{3}{|c|}{ Primeiro episódio } & \multicolumn{3}{|c|}{ Segundo episódio } & \multicolumn{3}{|c|}{ Terceiro episódio } \\
\hline & t (meses) & $\mathrm{PIO}(\mathrm{mmHg})$ & $\mathbf{H}$ & t (meses) & PIO (mmHg) & $\mathbf{H}$ & t (meses) & PIO (mmHg) & H \\
\hline OD & 2,5 & 3 & Cosopt $^{\circledR}$ & & & & & & \\
\hline OE & 4,0 & 0 & Cosopt ${ }^{\circledR}$ & 5,5 & 2 & Cosopt $^{\circledast}$ & 7,5 & 2 & $\begin{array}{c}\text { Azopt }^{\circledR}+ \\
\text { AlphaganP }\end{array}$ \\
\hline
\end{tabular}

$0 \mathrm{mmHg}$ a $3 \mathrm{mmHg}$, e o descolamento se resolveu apenas com suspensão dos colírios supracitados (Tabela 2).

O reconhecimento desta síndrome é fundamental para que o oftalmologista tome a medida mais eficaz para resolvê-la, que é a identificação e suspensão da droga responsável, além do uso tópico de corticosteróides e cicloplégicos. Este caso também ilustra a verdadeira saga que é, frequentemente, o pósoperatório da trabeculectomia e que não se deve desistir nunca enquanto houver alguma esperança.

\section{ABSTRACT}

A case of an 85 year-old white man with bilateral senile cataract and advanced primary open-angle glaucoma uncontrolled with maximal medical therapy, asteroid hyalosis in OD and age-related macular degeneration in OS, submitted to a phacotrabeculectomy OU with mitomycin-C is reported. Because the surgery failed in both eyes, even after laser suture lysis, 5-FU injections and needling, it was necessary to reintroduce hypotensive agents. With the fixed combination of $0.5 \%$ timolol $+2 \%$ dorzolamide, a serous choroidal detachment with marked hypotony developed in both eyes; with $1 \%$ brinzolamide it only occurred in the OS. The IOP raised and the choroidal detachment resolved completely after discontinuation of the medications. The dilemma was finally solved through repeat needling with subconjunctival 5-FU injections.

Keywords: Trabeculectomy/adverse effects; Mitomycin; Cho- roid diseases/etiology; Ocular hypotension; Recurrence; Case reports [Publication type]

\section{REFERÊNCIAS}

1. Rockwood EJ, Kalenak JW, Plotnik JL, Yoon JS, Sculley L, Medendorp SV. Prospective ultrasonographic evaluation of intraoperative and delayed postoperative suprachoroidal hemorrhage from glaucoma filtering surgery. J Glaucoma. 1995;4(1):16-24.

2. Burney EN, Quigley HA, Robin AL. Hypotony and choroidal detachment as late complications of trabeculectomy. Am J Ophthalmol. 1987;103(5):685-8.

3. Vela MA, Campbell DG. Hypotony and ciliochoroidal detachment following pharmacologic aqueous suppressant therapy in previously filtered patients. Ophthalmology. 1985;92(1):50-7.

4. Geyer O, Neudorfer M, Lazar M. Recurrent choroidal detachment following timolol therapy in previously filtered eye. Choroidal detachment post filtering surgery. Acta Ophthalmol (Copenh). 1992;70(5):702-3.

5. Berke SJ, Bellows AR, Shingleton BJ, Richter CU, Hutchinson BT. Chronic and recurrent choroidal detachment after glaucoma filtering surgery. Ophthalmology. 1987;94(2):154-62.

6. Fineman MS, Katz LJ, Wilson RP. Topical dorzolamide-induced hypotony and ciliochoroidal detachment in patients with previous filtration surgery. Arch Ophthalmol. 1996;114(8):1031-2.

7. Alimgil ML, Benian O. Choroidal effusion and shallowing of the anterior chamber after adjunctive therapy with latanoprost in a trabeculectomized patient with angle closure glaucoma. Int Ophthalmol. 2001;24(3):129-31.

8. Callahan C, Ayyala RS. Hypotony and choroidal effusion induced by topical timolol and dorzolamide in patients with previous glaucoma drainage device implantation. Ophthalmic Surg Lasers Imaging. 2003;34(6):467-9.

9. Silva MRBM, Jorge EN, Schellini SA. Descolamento ciliocoroidal e hipotonia causados por supressores do humor aquoso - síndrome da supersensibilidade aos supressores do humor aquoso. Arq Bras Oftalmol. 2000;63(1):83-6.

10. Goldberg S, Gallily R, Bishara S, Blumenthal EZ. Dorzolamide-induced choroidal detachment in a surgically untreated eye. Am J Ophthalmol. 2004;138(2): 285-6. 